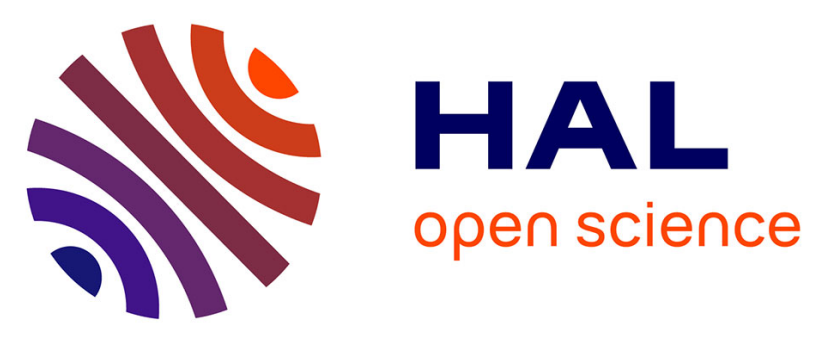

\title{
Activation of 1,2- and 1,3-Ketoamides with Thiourea Organocatalyst for the Enantioselective Domino Synthesis of Fuctionalized Cyclohexanes
}

Wilfried Raimondi, Maria del Mar Sanchez Duque, Sébastien Goudedranche, Adrien Quintard, Thierry Constantieux, Xavier Bugaut, Damien Bonne, Jean Rodriguez

\section{To cite this version:}

Wilfried Raimondi, Maria del Mar Sanchez Duque, Sébastien Goudedranche, Adrien Quintard, Thierry Constantieux, et al.. Activation of 1,2- and 1,3-Ketoamides with Thiourea Organocatalyst for the Enantioselective Domino Synthesis of Fuctionalized Cyclohexanes. Synthesis: Journal of Synthetic Organic Chemistry, 2013, 45, pp.1659-1666. 10.1055/s-0033-1338844 . hal-00861637

\section{HAL Id: hal-00861637 https://hal.science/hal-00861637}

Submitted on 16 Sep 2013

HAL is a multi-disciplinary open access archive for the deposit and dissemination of scientific research documents, whether they are published or not. The documents may come from teaching and research institutions in France or abroad, or from public or private research centers.
L'archive ouverte pluridisciplinaire HAL, est destinée au dépôt et à la diffusion de documents scientifiques de niveau recherche, publiés ou non, émanant des établissements d'enseignement et de recherche français ou étrangers, des laboratoires publics ou privés. 


\title{
Activation of 1,2- and 1,3-Ketoamides with Thiourea Organocatalyst for the Enantioselective Domino Synthesis of Functionalized Cyclohexanes
}

\author{
Wilfried Raimondi, ${ }^{\mathrm{a}}$ Maria del Mar Sanchez Duque, ${ }^{\mathrm{a}}$ Sébastien Goudedranche, ${ }^{\mathrm{a}}$ Adrien Quintard, ${ }^{\mathrm{a}}$ Thierry \\ Constantieux, ${ }^{\mathrm{a}}$ Xavier Bugaut, ${ }^{\mathrm{a}}{ }^{\mathrm{a}}$ Damien Bonne, ${ }^{* \mathrm{a}}$ Jean Rodriguez ${ }^{\mathrm{a}}$
}

${ }^{a}$ Aix Marseille Université, CNRS, iSm2 UMR 7313, 13397, Marseille, France.

Tel: +33 (0) 491288933 Fax: +33 (0) 491289187

E-mail: damien.bonne@univ-amu.fr or xavier.bugaut@univ-amu.fr

Received: The date will be inserted once the manuscript is accepted.

\begin{abstract}
Several reactive sites of 1,2- and 1,3-ketoamides were successively exploited in two complementary domino transformations for the synthesis of either polysubstituted mono cyclic or bridged bicyclic cyclohexanes with the creation of up to six stereogenic centers. In both cases, a chiral bifunctional thiourea organocatalyst allowed an efficient control of the chirality in the final carbocycles.
\end{abstract}

Keywords: Enantioselective organocatalysis, Domino reactions, Ketoamides, Cyclohexanes, Bifunctional thioureas

\section{Introduction}

The development of domino enantioselective organocatalytic methodologies has received an intense research attention attested by the high number of recent reports directed to the stereoselective construction of heterocylic and carbocyclic scaffolds. ${ }^{1}$ Among these, substituted chiral cyclohexanes are important building blocks in organic synthesis, ${ }^{2}$ and many of these approaches have allowed the control of the relative and absolute configurations in the construction of these versatile molecular architectures. ${ }^{3,4}$ These methods have the advantage of assembling efficiently the desired target from easily accessible starting materials along with the creation and control of multiple stereogenic carbon atoms. Most of these strategies employ simple substrates with multiple reactive sites that are involved successively in the formation of carbon-carbon and/or carbonheteroatom bonds. In this context, dicarbonyl compounds and more particularly ketoamides are attractive substrates given the high density of potential reactive sites and thus, they are well suited for designing new stereoselective domino transformations for the asymmetric synthesis of functionalized sixmembered carbocycles. ${ }^{5}$

\section{Results and discussion}

We have recently shown that 1,2- and 1,3-ketoamides 1 and 4 could be specifically activated using a thiourea-tertiary amine bifunctional organocatalyst and consequently, we successfully developed enantioselective Michael additions with these substrates and either nitroalkenes $\mathbf{2}$ or $\alpha, \beta$-unsaturated carbonyls 5 as the electrophilic partners. ${ }^{6}$ We now wish to exploit this work and the Michael adducts $\mathbf{3}$ and $\mathbf{6}$ as versatile synthetic platforms in subsequent transformations leading to more complex molecular frameworks, such as substituted cyclohexanes (Scheme 1).

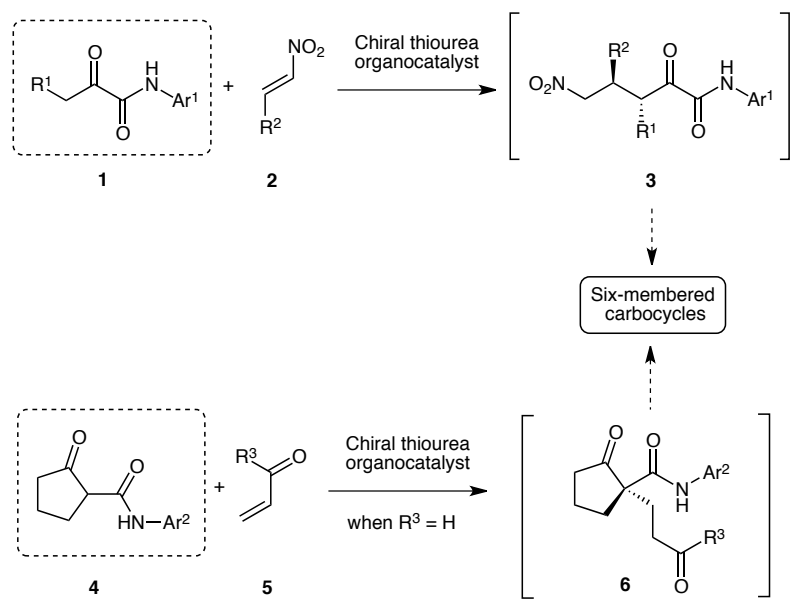

Scheme 1. Access to optically active six-membered carbocycles from 1,2- and 1,3-ketoamides $\mathbf{1}$ and $\mathbf{4}$

In the case of 1,2-ketoamides $\mathbf{1}$, and once the Michael adduct $\mathbf{3}$ is formed (Scheme 2), we reasoned that the addition of a second equivalent of nitroalkene could trigger another conjugate addition leading to intermediate 7. The latter should then undergo an intramolecular Henry reaction, terminating the domino transformation and affording the desired hexasubstituted cyclohexane $\mathbf{8}{ }^{7}$

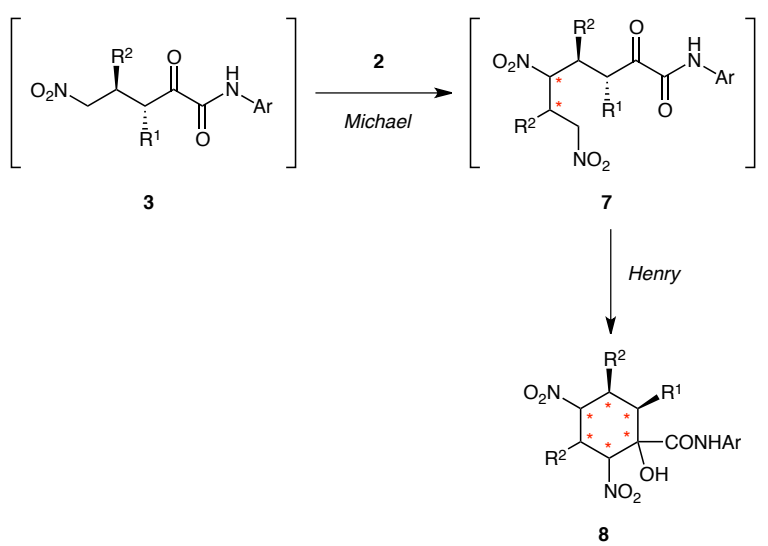

Scheme 2. Strategy for the synthesis of hexasubstituted cyclohexanes 8 We started our investigations by screening various bifunctional organocatalysts I-IV bearing either 
thiourea or squaramide hydrogen-bond donor subunits (Table 1). While catalyst I derived from cinchonine allowed only the formation of $\mathbf{8 a}$ as traces (entry 1, the sequence stopped at the Michael adduct stage), we were very pleased to find that Takemoto's catalyst II $(R, R \text {-TUC })^{8}$ was efficient for this domino transformation, affording 8a in good yield (67\%) with very good diastereo- and enantiomeric ratios $(>20: 1$ and 28:1 respectively, entry 2 ). Remarkably, one out of the 64 possible stereoisomers was obtained predominantly. The use of catalysts III and IV with a squaramide subunit gave poor results (entries 3 and 4) and only trace amounts of cyclohexane 8a were formed $(<5 \%)$. The highest yield and stereoselectivities were obtained in ethyl acetate (entry 2) and switching to dichloromethane led to the formation of the desired product in lower yield and selectivities (entry 5). Other solvents such as DMSO and MTBE were not suitable, leading to decomposition and poor reactivity, respectively. (entries 6 and 7).

Table 1. Optimization of the domino Michael-Michael-Henry ${ }^{a}$

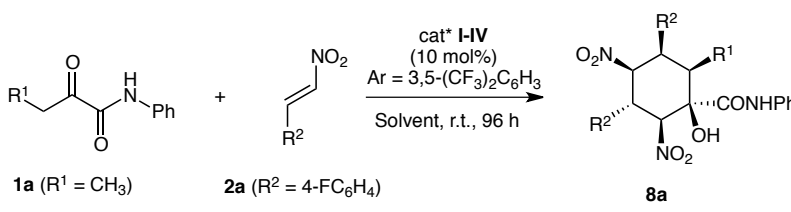<smiles>C=CC1CN2CCC1CC2C(NC(=S)NC)c1ccnc2ccccc12</smiles><smiles>CN(C)[C@@H]1CCCC[C@@H]1NC(=S)N[Al]</smiles><smiles>CNc1c(N[C@@H]2CCCC[C@H]2N(C)C)c(=O)c1=O</smiles>

III

\begin{tabular}{|c|c|c|c|c|c|}
\hline Entry & Catalyst & Solvent & Yield of $\mathbf{8 a}^{\mathrm{b}}$ & $\mathrm{dr}^{\mathrm{c}}$ & $\mathrm{er}^{\mathrm{d}}$ \\
\hline 1 & I & EtOAc & $<5 \%$ & n.d. & n.d. \\
\hline 2 & II & EtOAc & $62 \%$ & $>20: 1$ & $28: 1$ \\
\hline 3 & III & EtOAc & $<5 \%$ & n.d. & n.d. \\
\hline 4 & IV & EtOAc & $<5 \%$ & n.d. & n.d. \\
\hline 5 & II & $\mathrm{CH}_{2} \mathrm{Cl}_{2}$ & $45 \%$ & $1: 1.5$ & n.d. \\
\hline 6 & II & DMSO & decomposition & - & - \\
\hline 7 & II & MTBE & $<5 \%$ & n.d. & n.d. \\
\hline
\end{tabular}

${ }^{\mathrm{a}}$ All reactions were run using $0.2 \mathrm{mmol}$ of 1a, $0.42 \mathrm{mmol}$ of $2 \mathbf{a}$ in 0.4 $\mathrm{mL}$ of solvent. ${ }^{\mathrm{b}} \mathrm{Y}$ ields of analytically pure isolated products. ${ }^{c}$ Diastereomeric ratios were determined by ${ }^{1} \mathrm{H}$ NMR spectroscopy analysis of the crude reaction mixtures. ${ }^{\mathrm{d}}$ Enantiomeric ratio was determined by HPLC analysis on a chiral stationary phase.

After optimizing the reaction conditions, we studied the possibility of using different ketoamides $\mathbf{1}$ as well as different nitroalkenes $\mathbf{2}$ to synthesize various hexasubstituted cyclohexane derivatives 8a-e (Scheme 3). Hence, using Takemoto's catalyst II, in ethyl acetate at room temperature for four days, we were delighted to found that the cyclohexanes $\mathbf{8}$ were obtained in fair yields but most importantly with generally very good stereoselectivities. Electronwithdrawing as well as electron-donating groups on the aryl substituent of the ketoamide $\mathbf{1}$ were well tolerated in this reaction, although the use of ketoamide 1c $\left(\mathrm{R}^{1}=\mathrm{Et}, \mathrm{Ar}=2,5-\mathrm{Cl}_{2} \mathrm{C}_{6} \mathrm{H}_{3}\right)$ bearing a sterically demanding 2,5-dichlorophenyl substituent had a negative impact on the enantioselectivity of the domino reaction $(\mathbf{8 c}$, e.r. $=4: 1)$. Concerning the nitroalkene partner $\mathbf{2}$, both phenyl- and $p$ fluorophenylnitroalkenes are suitable substrates for this reaction.<smiles>[H][R]=C1CCCCC1NC(=S)Nc1cc(C(F)(F)F)cc(C(F)(F)F)c1</smiles>

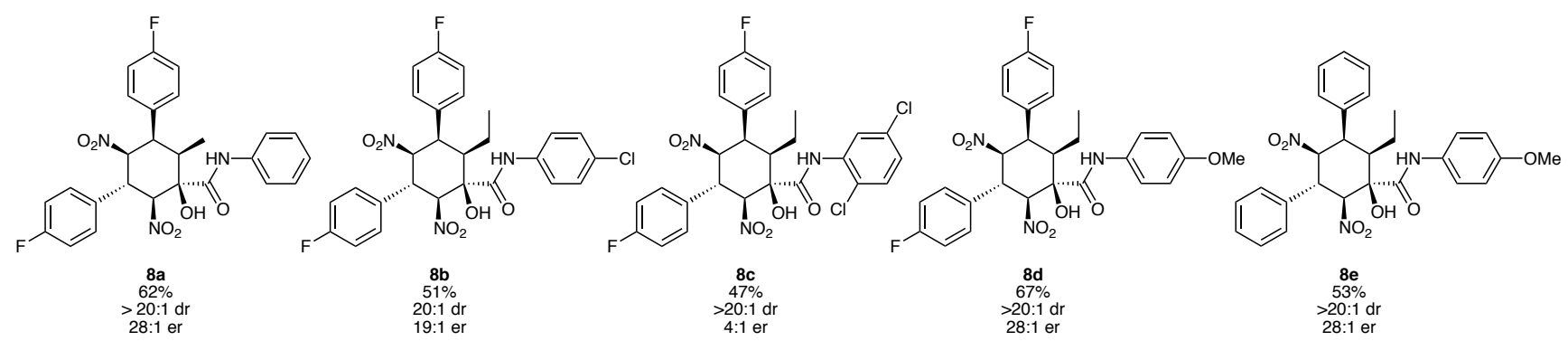


Our earlier studies had shown that Takemoto's catalyst II was also efficient to promote the enantioselective Michael addition of 1,3-ketoamides 4 to $\alpha, \beta$ unsaturated carbonyls. ${ }^{6 \mathrm{~b}}$ When acrolein 9 is used as the electrophilic reaction partner, two modes of cyclization can be envisaged for the initial adducts $\mathbf{6}$ (Scheme 4). The first one is the formation of hemiaminal 10, which we already exploited (path a). The second one may involve an intramolecular aldol reaction, leading directly to a bicyclo[3.2.1] octane 11 that bears a bridged six-membered ring with three stereogenic centers (path b). Given the prevalence of this skeleton in natural products and the challenge associated with their synthesis, ${ }^{9}$ new modular enantioselective accesses are of high synthetic interest.

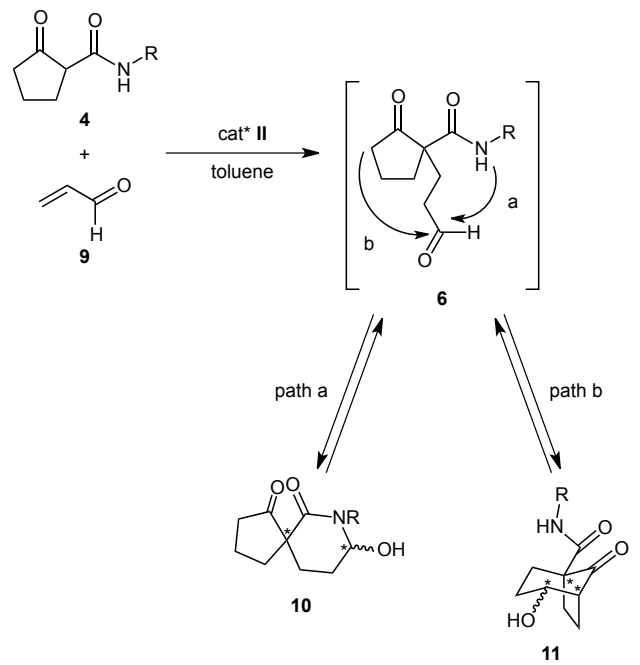

Scheme 4. Diverging pathways for the cyclization of the adducts of 1,3-ketoamides with acrolein

In our initial study with $N$-tosylketoamide, the hemiaminal was obtained as the sole product. $^{6 \mathrm{~b}}$ However, when we tried to extend this reactivity to $N$ arylketoamide $\mathbf{4 a}$, a spontaneous evolution towards a mixture of both products 10a and 11a was observed (Table 2), indicating that an equilibrium exists between compounds $\mathbf{1 0 a}$, which is the kinetic product, and 11a, which is the thermodynamic one. At $-20{ }^{\circ} \mathrm{C}$ in the presence of catalyst II, after $48 \mathrm{~h}$ at room temperature a 3.5:1 ratio between products $\mathbf{1 0 a}$ and 11a with the hydroxyl group in axial position was obtained (Entry 1). To favor the formation of the bicyclic product, different reaction conditions were investigated after the completion of the Michael addition. At room temperature, without the addition of any other reagent, a slow evolution was observed to reach a 1.5:1 ratio after an additional two days stirring (Entry 2). We could verify that Takemoto's catalyst II was really involved in this process as no interconversion between the products was obtained in its absence. As expected, increasing the temperature to $60{ }^{\circ} \mathrm{C}$ led to the formation of 11a as major compound with a 12:1 dr and a 4.7:1 er (Entry 3). We then looked for other additives that could affect the thermodynamic interconversion of 10a to 11a. While the addition of a catalytic amount of L-proline $\mathbf{V}$ had limited impact (Entry 4), stoichiometric amounts of $N$ heterocyclic carbene IPr VI (Entry 5) ${ }^{10}$ and 1,8diazabicyclo[5.4.0] undec-7-ene (DBU) VII (Entry 6) completely shifted the equilibrium towards the desired product 11a, albeit with very modest diastereoselectivities. In the last case, the er of the major diastereomer amounted to 5.1:1.

Table 2. Optimization of the reaction conditions for the synthesis of bicyclo[3.2.1] octane 11a $\mathbf{a}^{\mathrm{a}}$
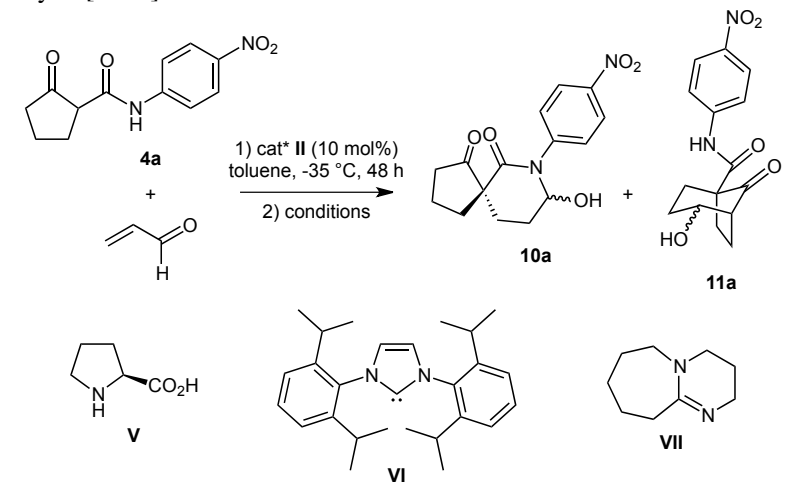

\begin{tabular}{|c|c|c|c|c|}
\hline Entry & conditions & $\begin{array}{c}\text { ratio } \\
\mathbf{1 0 a} / \mathbf{1 1 a} \mathbf{b}^{\mathrm{b}}\end{array}$ & $\begin{array}{c}\mathrm{dr} \\
\left(\mathrm{OH}_{\mathrm{ax}} / \mathrm{OH}_{\mathrm{eq}}\right)^{\mathrm{c}}\end{array}$ & $\mathrm{er}^{\mathrm{d}}$ \\
\hline 1 & $-^{\mathrm{e}}$ & $3.5: 1$ & $>20: 1$ & n.d. \\
\hline 2 & r.t., $2 \mathrm{~d}^{\mathrm{d}}$ & $1.5: 1$ & $>20: 1$ & n.d. \\
\hline 3 & $60^{\circ} \mathrm{C}, 2 \mathrm{~d}$ & $1: 9.3$ & $12: 1$ & $4.7: 1$ \\
\hline 4 & $\begin{array}{c}\mathbf{V}(20 \mathrm{~mol} \%), \\
\text { r.t., } 2 \mathrm{~d}\end{array}$ & $1: 1.1$ & $>20: 1$ & n.d. \\
\hline 5 & $\begin{array}{c}\text { VI (1.1 equiv), } \\
\text { r.t., } 3 \mathrm{~d}\end{array}$ & $<1: 20$ & $1: 2$ & n.d. \\
\hline 6 & $\begin{array}{c}\text { VII (1 equiv), } \\
\text { r.t., } 10 \text { min }\end{array}$ & $<1: 20^{\mathrm{f}}$ & $1.4: 1$ & $4.1: 1$ \\
& & & $3.7: 1$ \\
\hline
\end{tabular}

${ }^{\mathrm{a}}$ All reactions were run using $0.2 \mathrm{mmol}$ of $4 \mathbf{a}, 0.4 \mathrm{mmol}$ of 9 in $4 \mathrm{~mL}$ of solvent. ${ }^{b}$ Determined by ${ }^{1} \mathrm{H}$ NMR spectroscopy analysis of the crude reaction mixtures. ${ }^{\mathrm{c}}$ Diastereomeric ratios were determined by ${ }^{1} \mathrm{H}$ NMR spectroscopy analysis of the crude reaction mixtures. ${ }^{\mathrm{d}}$ Enantiomeric ratio of the major diastereomer of 11a $(\mathrm{OH}$ in axial position) was determined by HPLC analysis on a chiral stationary phase. ${ }^{\mathrm{e}}$ Analyses conducted directly at the end of the Michael addition step. ${ }^{\mathrm{f}} 82 \%$ yield after purification.

DBU was selected as the best promoter for the isomerization because it allowed an efficient reaction with a short reaction time and we investigated the behavior of other 1,3-ketoamides ${ }^{11}$ when submitted to these reaction conditions (Scheme 5). With other $\mathrm{N}$ aryl-1,3-ketoamides $\mathbf{4 b}$ and $\mathbf{4 c}$, we noticed that an evaporation of the excess acrolein was required prior to DBU addition in order to avoid a double Michael addition. Products 11b and 11c were obtained with reasonable yields but moderate stereoselectivities. Interestingly, $N$-aryl-1,3-ketoamides $\mathbf{4 d}$ could also be 
converted into the bicyclic product 11d. Because of the higher stability of the hemiaminal in this case, the isomerization with DBU had to be conducted at $60^{\circ} \mathrm{C}$. Once again, there was no diastereoselectivity but high enantioselectivities (66:1 and 14:1 er respectively) were obtained for both diastereomers. The differences in enantiomeric ratios observed between the different cyclization modes ${ }^{12}$ clearly indicate that these compounds are prone to racemization. As a result, particular care must be taken during the reaction and post functionalization to avoid such an issue.
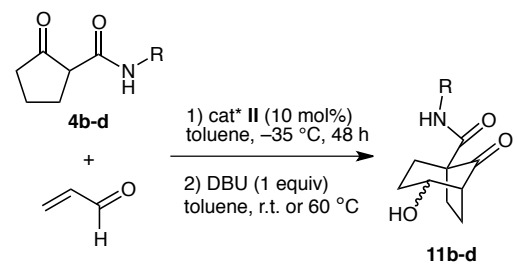
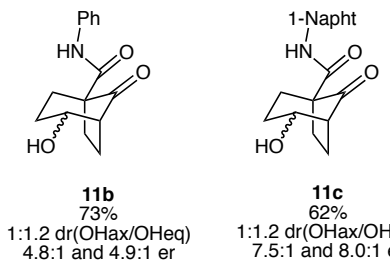

$62 \%$

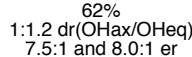

7.5:1 and 8.0:1 er
$11 \mathrm{~b}-\mathrm{d}$

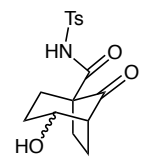

11d

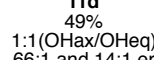

Scheme 5. Scope study of the formation of bicyclo[3.2.1] octanes 11

Bicyclic alcool 11d could also be derivatized with success by IBX oxidation in refluxing ethyl acetate, quantitatively generating

the bicyclo[3.2.1] octanedione 12 (Scheme 6). Most importantly, an excellent 24:1 er was observed for the formation of this valuable structure.

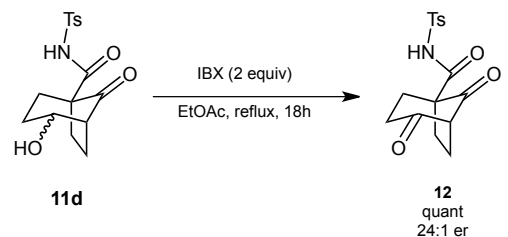

Scheme 6. Scope study of the formation of bicyclo[3.2.1] octanes $\mathbf{1 1}$

\section{Conclusion}

We have demonstrated that 1,2- and 1,3-ketoamides could be specifically activated with thiourea organocatalysts and used in domino transformations for the enantioselective synthesis of polysubstituted cyclohexanes. In the case of 1,2-ketoamides, we exploited both their pronucleophilic character, ${ }^{13}$ and the ketone moiety as electrophile site for the synthesis of hexasusbtituted cyclohexanes by reaction with nitroalkenes partners. Amazingly, one out of 64 stereoisomers was formed almost exclusively. Alternatively, 1,3-ketoamides where used as efficient $C$-bisnucleophiles with acroleine as the electrophilic partner allowing access to bicyclo[3.2.1]octanes in the optically active form with the formation of two new $C-C$ bonds and three stereogenic carbon atoms.
Further studies are currently undertaken in our laboratory in order to extend the scope of these promising cascade reactions.

\section{Experimental section}

All reagents were obtained from commercial sources and used as supplied unless otherwise stated. NMR data were recorded on a Bruker Avance 400 spectrometer in $\mathrm{CDCl}_{3}$ and chemical shifts $(\delta)$ are given in ppm relative to the residual non-deutered solvent signal for ${ }^{1} \mathrm{H} \mathrm{NMR}\left(\mathrm{CHCl}_{3}: 7.26 \mathrm{ppm}\right)$, and relative to the deutered solvent signal for ${ }^{13} \mathrm{C}$ NMR $\left(\mathrm{CDCl}_{3}: 77.16 \mathrm{ppm}\right)$; coupling constants $(J)$ are in Hertz, and the classical abbreviations are used to describe the signal multiplicity. High-resolution mass spectra were obtained from the Spectropole (http://www.spectropole.u-3mrs.fr/). Optical rotations were measured with a PERKIN ELMER 241 micropolarimeter. Melting points (mp) were determined with a Büchi Melting-point B-450 apparatus and were not corrected. Thin layer chromatography (TLCs) were developed on silica Merck $60 F_{254}$. Visualization was achieved under a UVP mineralight UVGL-58 lamp, and by developing the plates with phosphomolybdic acid and $p$ anisaldehyde reagents. The products were purified by flash column chromatography on silica gel 60 (Merck 1.09386.9025, 230-400 mesh).

\section{General procedure for the synthesis of cyclohexanes 8}

1,2-ketoamides 1 ( 1.00 equiv), nitroalkenes 2 (2.10 equiv) and thiourea-catalyst II (0.10 equiv) were successively added as solid in a sealed tube and dissolved in ethyl acetate $(0.5 \mathrm{M})$. After 96 hours of reaction time at room temperature, the solvent was evaporated and the crude product was purified by flash chromatography on silica gel (eluent: ethyl acetate/petroleum ether, 30:70 then 50:50). The diastereomeric ratio (dr) was determined by ${ }^{1} \mathrm{H}$ NMR spectroscopy analysis of the crude product and the enantiomeric ratio (er) was determined by HPLC analysis on a chiral stationary phase.

8a: this product was synthesized starting from $1 \mathrm{a}\left(\mathrm{R}^{1}\right.$ $=\mathrm{Me}, \mathrm{Ar}=\mathrm{Ph}, 36 \mathrm{mg}, 0.2 \mathrm{mmol})$ and $\mathbf{2 a}\left(\mathrm{R}^{2}=4-\right.$ $\mathrm{FC}_{6} \mathrm{H}_{4}, 70 \mathrm{mg}, 0.42 \mathrm{mmol}$ ) according to the above procedure and was obtained as a white solid $(63 \mathrm{mg}$, $62 \%) . \mathrm{R} f(\mathrm{EA} / \mathrm{PE}, 3: 7)=0.30 ; \mathrm{mp}=181-182{ }^{\circ} \mathrm{C} ; \alpha_{\mathrm{D}}{ }^{30}$ $\left(\mathrm{CH}_{2} \mathrm{Cl}_{2}, c=1.0\right)=-22.3$; Chiral HPLC (ChiralPak AD-H, hexane $/ \mathrm{iPrOH}=9: 1$, flow rate $=1.0 \mathrm{~mL} / \mathrm{min}$, $\lambda=306 \mathrm{~nm}): \mathrm{t}_{\text {minor }}=24.7 \mathrm{~min}, \mathrm{t}_{\text {major }}=28.0 \mathrm{~min}, \mathrm{er}=$ 99:1; ${ }^{1} \mathrm{H}$ NMR (400 MHz, $\mathrm{CD}_{2} \mathrm{Cl}_{2}$ ): $\delta 8.65$ (brs, $1 \mathrm{H}$, $\mathrm{NH}), 7.62(\mathrm{br} \mathrm{s}, 2 \mathrm{H}), 7.52(\mathrm{~d}, J=8.0 \mathrm{~Hz}, 2 \mathrm{H}), 7.42-$ $7.36(\mathrm{~m}, 4 \mathrm{H}), 7.20(\mathrm{~d}, J=8.0 \mathrm{~Hz}, 1 \mathrm{H}), 7.08(\mathrm{~d}, J=8.0$ $\mathrm{Hz}, 2 \mathrm{H}), 7.02(\mathrm{~d}, J=8.0 \mathrm{~Hz}, 2 \mathrm{H}, \mathrm{ArH}), 5.76(\mathrm{~d}, J=$ $12.0 \mathrm{~Hz}, 1 \mathrm{H}), 5.60(\mathrm{dd}, J=12.0,7.2 \mathrm{~Hz}, 1 \mathrm{H}), 4.61$ (dd, $J=12.0,12.0 \mathrm{~Hz}, 1 \mathrm{H}), 4.61(\mathrm{~s}, 1 \mathrm{H}, \mathrm{OH}), 4.00$ 
(dd, $J=7.2,7.2 \mathrm{~Hz}, 1 \mathrm{H}), 3.05-2.99(\mathrm{~m}, 1 \mathrm{H}), 0.98$ (d, $J=7.2 \mathrm{~Hz}, 3 \mathrm{H}) ;{ }^{13} \mathrm{C}$ NMR $\left(100 \mathrm{MHz}, \mathrm{CD}_{2} \mathrm{Cl}_{2}\right): \delta$ $169.1,163.4(\mathrm{~d}, J=248 \mathrm{~Hz}), 163.3(\mathrm{~d}, J=248 \mathrm{~Hz})$, $138.8(\mathrm{~d}, J=8 \mathrm{~Hz}, 2 \mathrm{C}), 136.7$ (2C), 130.5 (d, $J=8$ $\mathrm{Hz}, 2 \mathrm{C}), 129.9$ (d, $J=4 \mathrm{~Hz}), 129.7,129.5$ (d, $J=4$ $\mathrm{Hz}$, 126.1, 120.7 (2C), 116.8 (d, $J=17 \mathrm{~Hz}, 2 \mathrm{C})$, 115.7 (d, $J=17 \mathrm{~Hz}, 2 \mathrm{C}), 92.0,89.3,80.1,49.7,42.2$, 39.3, 14.0; HRMS (ES+): $\mathrm{m} / \mathrm{z}$ calcd for $\left[\left(\mathrm{C}_{26} \mathrm{H}_{24} \mathrm{~F}_{2} \mathrm{~N}_{3} \mathrm{O}_{6}+\mathrm{H}\right)^{+}\right]$: 512.1628; found: 512.1619 .

8b: this product was synthesized starting from $\mathbf{1 b}\left(\mathrm{R}^{1}\right.$ $\left.=\mathrm{Et}, \mathrm{Ar}=4-\mathrm{ClC}_{6} \mathrm{H}_{4}, 45 \mathrm{mg}, 0.2 \mathrm{mmol}\right)$ and $\mathbf{2 a}\left(\mathrm{R}^{2}=\right.$ $\left.4-\mathrm{FC}_{6} \mathrm{H}_{4}, 70 \mathrm{mg}, 0.42 \mathrm{mmol}\right)$ according to the above procedure and was obtained as a white solid (57 mg, $51 \%$ ). $\mathrm{mp} 248^{\circ} \mathrm{C}$ (dec.). $\mathrm{R} f(\mathrm{EA} / \mathrm{PE}, 1: 4)=0.30$; Chiral HPLC (ChiralPak IA, hexane/ethanol/chloroforme = $5: 3: 2$, flow rate $=1.0 \mathrm{~mL} / \mathrm{min}, \lambda=254 \mathrm{~nm}): t_{\text {minor }}=$ $4.0 \mathrm{~min}, \mathrm{t}_{\mathrm{major}}=5.8 \mathrm{~min}$, er $=19: 1 ;{ }^{1} \mathrm{H}$ NMR $(400$ $\mathrm{MHz}, \mathrm{CD}_{2} \mathrm{Cl}_{2}$ ): $\delta 8.68$ (brs, $1 \mathrm{H}, \mathrm{NH}$ ), 7.70 (br s, 2H), $7.50(\mathrm{~d}, J=8.9 \mathrm{~Hz}, 2 \mathrm{H}), 7.37-7.31(\mathrm{~m}, 4 \mathrm{H}), 7.08(\mathrm{~d}, J$ $=8.9 \mathrm{~Hz}, 2 \mathrm{H}), 7.03(\mathrm{~d}, J=8.9 \mathrm{~Hz}, 2 \mathrm{H}), 5.61(\mathrm{~d}, J=$ $12.5 \mathrm{~Hz}, 1 \mathrm{H}), 5.43$ (dd, $J=12.5,6.9 \mathrm{~Hz}, 1 \mathrm{H}), 4.65$ (s, $1 \mathrm{H}, \mathrm{OH}), 4.45(\mathrm{dd}, J=12.5,12.5 \mathrm{~Hz}, 1 \mathrm{H}), 4.16(\mathrm{dd}, J$ $=6.9,6.9 \mathrm{~Hz}, 1 \mathrm{H}), 2.75-2.70(\mathrm{~m}, 1 \mathrm{H}), 1.44-1.33(\mathrm{~m}$, $2 \mathrm{H}), 0.87(\mathrm{~d}, J=7.3 \mathrm{~Hz}, 3 \mathrm{H}) ;{ }^{13} \mathrm{C}$ NMR $(100 \mathrm{MHz}$, $\left.\mathrm{CD}_{2} \mathrm{Cl}_{2}\right): \delta 169.3,163.2(\mathrm{~d}, J=248 \mathrm{~Hz}), 163.1(\mathrm{~d}, J=$ $248 \mathrm{~Hz}), 135.3,133.8$ (d, $J=8 \mathrm{~Hz}, 2 \mathrm{C}), 130.9,130.4$ (d, $J=8 \mathrm{~Hz}, 2 \mathrm{C}), 129.7$ (d, $J=4 \mathrm{~Hz}), 129.6$ (2C), $129.0(\mathrm{~d}, J=4 \mathrm{~Hz}), 121.9(2 \mathrm{C}), 116.7$ (d, $J=22 \mathrm{~Hz}$, 2C), $115.8(\mathrm{~d}, J=22 \mathrm{~Hz}, 2 \mathrm{C}), 92.1,89.8,80.2,46.7$, 46.1, 41.6, 20.8, 12.0; $\mathrm{MS}(\mathrm{ES}+): \mathrm{m} / \mathrm{z}\left[(\mathrm{M}+\mathrm{Na})^{+}\right]$582; HRMS (ES+): $\mathrm{m} / \mathrm{z}$ calcd for $\left[\left(\mathrm{C}_{27} \mathrm{H}_{24} \mathrm{~N}_{3} \mathrm{O}_{6} \mathrm{ClF}_{2}+\mathrm{H}\right)^{+}\right]$: 560.1394; found: 560.1395 .

8c: this product was synthesized starting from 1c $\left(\mathrm{R}^{1}\right.$ $\left.=\mathrm{Et}, \mathrm{Ar}=2,5-\mathrm{Cl}_{2} \mathrm{C}_{6} \mathrm{H}_{3}, 52 \mathrm{mg}, 0.2 \mathrm{mmol}\right)$ and $\mathbf{2 a}\left(\mathrm{R}^{2}\right.$ $=4-\mathrm{FC}_{6} \mathrm{H}_{4}, 70 \mathrm{mg}, 0.42 \mathrm{mmol}$ ) according to the above procedure and was obtained as a white solid $(57 \mathrm{mg}$, $47 \%)$. R $f($ EA/PE, $1: 4)=0.41 ; \mathrm{mp}=203-205^{\circ} \mathrm{C} ; \alpha_{\mathrm{D}}{ }^{22}$ $\left(\mathrm{CH}_{2} \mathrm{Cl}_{2}, c=0.52\right)=-12.9$; Chiral HPLC (ChiralPak IA, hexane/ethanol/chloroforme $=3: 1: 1$, flow rate $=$ $1.0 \mathrm{~mL} / \mathrm{min}, \lambda=254 \mathrm{~nm}): \mathrm{t}_{\text {minor }}=4.1 \mathrm{~min}, \mathrm{t}_{\text {major }}=5.8$ min, er $=4: 1 ;{ }^{1} \mathrm{H}$ NMR $\left(400 \mathrm{MHz}, \mathrm{CD}_{2} \mathrm{Cl}_{2}\right): \delta 9.21$ (brs, $1 \mathrm{H}, \mathrm{NH}), 8.35$ (brs, 1H), 7.72-7.69 (m, 2H), 7.38-7.34 (m, 4H), 7.14-7.04 (m, 5H), $5.56(\mathrm{~d}, J=$ $12.5 \mathrm{~Hz}, 1 \mathrm{H}), 5.39$ (dd, $J=12.5,6.8 \mathrm{~Hz}, 1 \mathrm{H}), 4.72$ (s, $1 \mathrm{H}, \mathrm{OH}), 4.46(\mathrm{dd}, J=12.5,12.5 \mathrm{~Hz}, 1 \mathrm{H}), 4.17(\mathrm{dd}, J$ $=6.8,6.8 \mathrm{~Hz}, 1 \mathrm{H}), 2.72-2.67(\mathrm{~m}, 1 \mathrm{H}), 1.55-1.37(\mathrm{~m}$, $2 \mathrm{H}), 0.88(\mathrm{~d}, J=7.3 \mathrm{~Hz}, 3 \mathrm{H}) ;{ }^{13} \mathrm{C}$ NMR $(100 \mathrm{MHz}$, $\left.\mathrm{CD}_{2} \mathrm{Cl}_{2}\right): \delta 169.5,163.3(\mathrm{~d}, J=248 \mathrm{~Hz}), 163.2(\mathrm{~d}, J=$ $248 \mathrm{~Hz}$ ), 134.4, 133.8 (d, $J=8 \mathrm{~Hz}, 2 \mathrm{C}), 130.5$ (2C), $130.4(\mathrm{~d}, J=8 \mathrm{~Hz}), 129.6(\mathrm{~d}, J=4 \mathrm{~Hz}), 128.9(\mathrm{~d}, J=$ $4 \mathrm{~Hz}), 126.4,122.6,121.8,116.7$ (d, $J=22 \mathrm{~Hz}, 2 \mathrm{C})$, $115.9(\mathrm{~d}, J=22 \mathrm{~Hz}, 2 \mathrm{C}), 92.1,89.8,80.5,46.7,46.2$, 41.6, 20.7, 12.0; $\mathrm{MS}(\mathrm{ES}+): \mathrm{m} / \mathrm{z}\left[(\mathrm{M}+\mathrm{Na})^{+}\right]$616; HRMS (ES+): $\mathrm{m} / \mathrm{z}$ calcd for $\left[\left(\mathrm{C}_{27} \mathrm{H}_{23} \mathrm{~N}_{3} \mathrm{O}_{6} \mathrm{Cl}_{2} \mathrm{~F}_{2}+\mathrm{H}\right)^{+}\right]$: 594.1005; found: 594.1003.

8d: this product was synthesized starting from $\mathbf{1 d}\left(\mathrm{R}^{1}\right.$ $\left.=\mathrm{Et}, \mathrm{Ar}=4-\mathrm{MeOC}_{6} \mathrm{H}_{4}, 44 \mathrm{mg}, 0.2 \mathrm{mmol}\right)$ and $\mathbf{2 a}\left(\mathrm{R}^{2}\right.$ $=4-\mathrm{FC}_{6} \mathrm{H}_{4}, 70 \mathrm{mg}, 0.42 \mathrm{mmol}$ ) according to the above procedure and was obtained as a white solid $(74 \mathrm{mg}$, $67 \%) . \mathrm{R} f(\mathrm{EA} / \mathrm{PE}, 1: 4)=0.11 ; \mathrm{mp}=109-111^{\circ} \mathrm{C} ; \alpha_{\mathrm{D}}{ }^{30}$ $\left(\mathrm{CH}_{2} \mathrm{Cl}_{2}, 0.57\right)=+29.5$; Chiral HPLC (ChiralPak IB, hexane/ethanol $=4: 1$, flow rate $=1.0 \mathrm{~mL} / \mathrm{min}, \lambda=254$ $\mathrm{nm}): \mathrm{t}_{\text {minor }}=10.9 \mathrm{~min}, \mathrm{t}_{\text {major }}=6.6 \mathrm{~min}$, er $=28: 1 ;{ }^{1} \mathrm{H}$ NMR (400 MHz, $\mathrm{CD}_{2} \mathrm{Cl}_{2}$ ): $\delta 8.60$ (brs, $1 \mathrm{H}, \mathrm{NH}$ ), 7.69-7.66 (m, 2H), $7.41(\mathrm{~d}, J=9.0 \mathrm{~Hz}, 2 \mathrm{H}), 7.34-7.30$ $(\mathrm{m}, 2 \mathrm{H}), 7.05(\mathrm{~d}, J=9.0 \mathrm{~Hz}, 2 \mathrm{H}), 6.96(\mathrm{~d}, J=9.0 \mathrm{~Hz}$, $2 \mathrm{H}), 6.88(\mathrm{~d}, J=9.0 \mathrm{~Hz}, 2 \mathrm{H}), 5.70(\mathrm{~d}, J=12.3 \mathrm{~Hz}$, $1 \mathrm{H}), 5.50(\mathrm{dd}, J=12.3,6.9 \mathrm{~Hz}, 1 \mathrm{H}), 4.69(\mathrm{~s}, 1 \mathrm{H}, \mathrm{OH})$, $4.41(\mathrm{dd}, J=12.3,12.3 \mathrm{~Hz}, 1 \mathrm{H}), 4.11(\mathrm{dd}, J=6.9,6.9$ $\mathrm{Hz}, 1 \mathrm{H}), 2.82-2.76(\mathrm{~m}, 1 \mathrm{H}), 1.47-1.36(\mathrm{~m}, 2 \mathrm{H}), 0.87$ $(\mathrm{d}, J=7.3 \mathrm{~Hz}, 3 \mathrm{H}) ;{ }^{13} \mathrm{C}$ NMR $\left(100 \mathrm{MHz}, \mathrm{CD}_{2} \mathrm{Cl}_{2}\right): \delta$ $168.7,162.8$ (d, $J=248 \mathrm{~Hz}), 162.7$ (d, $J=248 \mathrm{~Hz})$, 157.5, $133.2(\mathrm{~d}, J=8 \mathrm{~Hz}, 2 \mathrm{C}), 129.9$ (d, $J=8 \mathrm{~Hz}$, 2C), $129.0(\mathrm{~d}, J=4 \mathrm{~Hz}), 128.9,128.5$ (d, $J=4 \mathrm{~Hz})$, 122.0 (2C), 116.3 (d, $J=22 \mathrm{~Hz}, 2 \mathrm{C}), 115.6$ (d, $J=22$ $\mathrm{Hz}, 2 \mathrm{C}), 114.4$ (2C), 91.7, 89.3, 79.7, 55.5, 46.4, 45.5, 41.2, 20.3, 11.8; $\mathrm{MS}(\mathrm{ES}+): \mathrm{m} / \mathrm{z}\left[(\mathrm{M}+\mathrm{Na})^{+}\right] 579$; HRMS (ES+): $\mathrm{m} / \mathrm{z}$ calcd for $\left[\left(\mathrm{C}_{28} \mathrm{H}_{27} \mathrm{~N}_{3} \mathrm{O}_{7} \mathrm{~F}_{2}+\mathrm{H}\right)^{+}\right]$: 556.1890; found: 556.1889.

8e: this product was synthesized starting from $\mathbf{1 d}\left(\mathrm{R}^{1}\right.$ $\left.=\mathrm{Et}, \mathrm{Ar}=4-\mathrm{MeOC}_{6} \mathrm{H}_{4}, 44 \mathrm{mg}, 0.2 \mathrm{mmol}\right)$ and $\mathbf{2} \mathbf{b}\left(\mathrm{R}^{2}\right.$ $\left.=\mathrm{C}_{6} \mathrm{H}_{5}, 63 \mathrm{mg}, 0.42 \mathrm{mmol}\right)$ according to the above procedure and was obtained as a white solid $(55 \mathrm{mg}$, $53 \%) . \mathrm{R} f(\mathrm{EtOAc} / \mathrm{PE}, 1: 4)=0.18 ; \mathrm{mp}=139-141{ }^{\circ} \mathrm{C}$; $\alpha_{\mathrm{D}}{ }^{22}\left(\mathrm{CH}_{2} \mathrm{Cl}_{2}, \quad c=9.9\right)=+9.7$; Chiral HPLC (ChiralPak IB, exane/ethanol $=7: 3$, flow rate $=1.0$ $\mathrm{mL} / \mathrm{min}, \lambda=254 \mathrm{~nm}): \mathrm{t}_{\text {minor }}=6.6 \mathrm{~min}, \mathrm{t}_{\text {major }}=5.3 \mathrm{~min}$, $\mathrm{er}=28: 1 ;{ }^{1} \mathrm{H}$ NMR $\left(400 \mathrm{MHz}, \mathrm{CDCl}_{3}\right): \delta 8.59$ (brs, $1 \mathrm{H}, \mathrm{NH}), 7.69$ (br s, 2H), 7.40-7.36 (m, 8H), 7.34$7.28(\mathrm{~m}, 2 \mathrm{H}), 6.86(\mathrm{~d}, J=9.0 \mathrm{~Hz}, 2 \mathrm{H}), 5.69(\mathrm{~d}, J=$ $12.5 \mathrm{~Hz}, 1 \mathrm{H}), 5.48(\mathrm{dd}, J=12.5,6.7 \mathrm{~Hz}, 1 \mathrm{H}), 4.54$ (dd, $J=12.5,12.5 \mathrm{~Hz}, 1 \mathrm{H}), 4.52(\mathrm{~s}, 1 \mathrm{H}, \mathrm{OH}), 4.13$ $(\mathrm{dd}, J=6.7,6.7 \mathrm{~Hz}, 1 \mathrm{H}), 3.77(\mathrm{~s}, 3 \mathrm{H}), 2.81-2.76(\mathrm{~m}$, 1H), $1.45-1.37(\mathrm{~m}, 2 \mathrm{H}), 0.91(\mathrm{~d}, J=7.3 \mathrm{~Hz}, 3 \mathrm{H}) ;{ }^{13} \mathrm{C}$ NMR $\left(100 \mathrm{MHz}, \mathrm{CDCl}_{3}\right): \delta 168.8,157.3,133.7$, 132.9 (2C), 131.3 (2C), 129.2 (2C), $129.0,128.7$ (2C), 128.6, 128.2 (2C), 122.0 (2C), 114.3 (2C), 92.0, 89.4, 79.7, 55.5, 47.0, 45.7, 41.9, 20.4, 11.9. MS (ES+): m/z $\left[(\mathrm{M}+\mathrm{Na})^{+}\right] \quad 542 ;$ HRMS $(\mathrm{ES}+): \mathrm{m} / \mathrm{z}$ calcd for $\left[\left(\mathrm{C}_{28} \mathrm{H}_{29} \mathrm{~N}_{3} \mathrm{O}_{7}+\mathrm{H}\right)^{+}\right]$: 520.2078; found: 520.2077.

\section{General procedure for the synthesis of bicyclo[3.2.1]octanes 11}

A solution of 1,3-ketoamides 4 ( $0.2 \mathrm{mmol}, 1$ equiv $)$ and thiourea-catalyst II $(0.02 \mathrm{mmol}, 0.1$ equiv. $)$ in dry toluene $(4 \mathrm{~mL})$ was cooled at $-35^{\circ} \mathrm{C}$. Acrolein 9 (27 $\mu \mathrm{L}, 0.4 \mathrm{mmol}, 2$ equiv) was added and the mixture was stirred for 24-48 hours until full conversion of 4 . Then DBU ( $30 \mu \mathrm{L}, 0.2 \mathrm{mmol}, 1$ equiv.) was added to the reaction medium, which was subsequently warmed to room temperature and stirred for 1 hour. The mixture was concentrated under reduced pressure. $\mathrm{HCl}(1 \mathrm{~N})$ was added and the mixture was extracted twice with $\mathrm{CH}_{2} \mathrm{Cl}_{2}$. The combined organic phase was washed with brine, dried over anhydrous $\mathrm{Na}_{2} \mathrm{SO}_{4}$ and evaporated under reduced pressure. The crude product 
was purified by flash column chromatography (ethyl acetate/petroleum ether) to afford pure $\mathbf{1 1 .}$

11a: this product was synthesized starting from $4 a(R$ $=4-\mathrm{NO}_{2} \mathrm{C}_{6} \mathrm{H}_{4}, 50 \mathrm{mg}, 0.2 \mathrm{mmol}$ ) according to the above procedure and was obtained as a 1.4:1 mixture of diastereomers as a pale yellow solid (50 mg, 82\%). $\mathrm{R} f\left(\mathrm{EA} / \mathrm{CH}_{2} \mathrm{Cl}_{2}, 2: 3\right)=0.33 ; \mathrm{mp}=137-139^{\circ} \mathrm{C}$; Chiral HPLC (Lux-Amylose-2, hexane $/ \mathrm{PrOH}=7: 3$, flow rate $=1.0 \mathrm{~mL} / \mathrm{min}, \lambda=220 \mathrm{~nm})$ : diastereomer $\mathrm{OH}_{\mathrm{ax}}$ : $\mathrm{t}_{\text {minor }}=18.0 \mathrm{~min}, \mathrm{t}_{\text {major }}=21.0 \mathrm{~min}$, er $=4.1: 1$, diastereomer $\mathrm{OH}_{\mathrm{eq}}: \mathrm{t}_{\text {minor }}=10.1 \mathrm{~min}, \mathrm{t}_{\text {major }}=11.3 \mathrm{~min}$, er $=3.7: 1 ;{ }^{1} \mathrm{H}$ NMR $\left(400 \mathrm{MHz}, \mathrm{CDCl}_{3}\right)$ : diastereomer $\mathrm{OH}_{\mathrm{ax}}: \delta 10.21(\mathrm{~s}, \mathrm{NH}), 8.21(\mathrm{~d}, J=9.2 \mathrm{~Hz}, 2 \mathrm{H}), 7.77$ (d, $J=9.2 \mathrm{~Hz}, 2 \mathrm{H}), 4.45$ (s, 1H), 2.67 (dd, $J=7.1,5.4$ $\mathrm{Hz}, 1 \mathrm{H}), 2.46-2.40$ (m, 1H), 2.39-2.31 (m, 2H), 2.31$2.12(\mathrm{~m}, 2 \mathrm{H}), 2.03-1.92(\mathrm{~m}, 1 \mathrm{H}), 1.84-1.71(\mathrm{~m}, 2 \mathrm{H})$, diastereomer $\mathrm{OH}_{\mathrm{eq}}: \delta 10.07(\mathrm{~s}, \mathrm{NH}), 8.21(\mathrm{~d}, J=9.2$ $\mathrm{Hz}, 2 \mathrm{H}), 7.77$ (d, $J=9.2 \mathrm{~Hz}, 2 \mathrm{H}), 4.14$ (br s, 1H), 2.75 (dd, $J=7.0,3.1 \mathrm{~Hz}, 1 \mathrm{H}), 2.46-2.40(\mathrm{~m}, 1 \mathrm{H}), 2.39-2.31$ (m, 2H), 2.31-2.12 (m, 2H), 2.03-1.92 (m, 1H), 1.84$1.71(\mathrm{~m}, 2 \mathrm{H}) .{ }^{13} \mathrm{C}$ NMR (75 MHz, DMSO-d6): diastereomer $\mathrm{OH}_{\mathrm{ax}}: \delta 213.0,171.0,145.42,142.21$, 124.88 (2C), 119.3 (2C), 76.9, 57.5, 52.3, 34.1, 26.6, $25.4,18.9$, diastereomer $\mathrm{OH}_{\mathrm{eq}}: \delta 213.1,170.7,145.36$, 142.23, 124.94 (2C), 119.2 (2C), 73.6, 57.7, 54.8, 31.8, 28.2, 26.4, 16.3; MS (ES+): $\mathrm{m} / \mathrm{z}\left[(\mathrm{M}+\mathrm{Na})^{+}\right] 327$; HRMS (ES+): $\mathrm{m} / \mathrm{z}$ calcd for $\left[\left(\mathrm{C}_{15} \mathrm{H}_{16} \mathrm{~N}_{2} \mathrm{O}_{5}+\mathrm{H}\right)^{+}\right]$: 305.1132; found: 305.1130 .

11b: this product was synthesized starting from $4 b(R$ $\left.=\mathrm{C}_{6} \mathrm{H}_{5}, 41 \mathrm{mg}, 0.2 \mathrm{mmol}\right)$ by adapting the above procedure. After full conversion of $\mathbf{4 b}$, the excess of acrolein was eliminated by reduced pressure (100 mbar), without evaporation of toluene. Then DBU was added and the reaction mixture was stirred at room temperature for 1 hour to afford $\mathbf{1 1 b}$ as a $1: 1.2$ mixture of diastereomers as a colorless viscous liquid (38 mg, 73\%). $\mathrm{R} f\left(\mathrm{EA} / \mathrm{CH}_{2} \mathrm{Cl}_{2}, 2: 3\right)=0.28$; Chiral HPLC (Chiralcel OD-3, hexane $/ \mathrm{iPrOH}=9: 1$, flow rate $=1.0 \mathrm{~mL} / \mathrm{min}, \lambda=254 \mathrm{~nm})$ : diastereomer $\mathrm{OH}_{\mathrm{ax}}$ : $\mathrm{t}_{\text {minor }}=18.9 \mathrm{~min}, \mathrm{t}_{\text {major }}=23.0 \mathrm{~min}$, er $=4.8: 1$, diastereomer $\mathrm{OH}_{\mathrm{eq}}: \mathrm{t}_{\text {minor }}=17.0 \mathrm{~min}, \mathrm{t}_{\text {major }}=20.4 \mathrm{~min}$, er $=4.9: 1 ;{ }^{1} \mathrm{H} \mathrm{NMR}\left(400 \mathrm{MHz}, \mathrm{CDCl}_{3}\right)$ : diastereomer $\mathrm{OH}_{\mathrm{ax}}: \delta 9.79(\mathrm{~s}, \mathrm{NH}), 7.60-7.51(\mathrm{~m}, 2 \mathrm{H}), 7.32(\mathrm{t}, J=$ $7.9 \mathrm{~Hz}, 2 \mathrm{H}), 7.10$ (td, $J=7.3,1.2 \mathrm{~Hz}, 1 \mathrm{H}), 4.42-4.30$ $(\mathrm{m}, 1 \mathrm{H}), 2.64-2.57(\mathrm{~m}, 1 \mathrm{H}), 2.44-2.19(\mathrm{~m}, 4 \mathrm{H}), 2.16-$ $1.99(\mathrm{~m}, 1 \mathrm{H}), 1.96-1.64(\mathrm{~m}, 3 \mathrm{H})$, diastereomer $\mathrm{OH}_{\mathrm{eq}}$ :

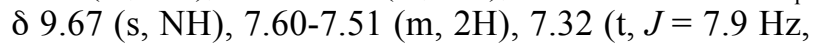
$2 \mathrm{H}), 7.10(\mathrm{td}, J=7.3,1.2 \mathrm{~Hz}, 1 \mathrm{H}), 4.18-4.03(\mathrm{~m}, 1 \mathrm{H})$, $2.72(\mathrm{dd}, J=6.9,3.2 \mathrm{~Hz}, 1 \mathrm{H}), 2.44-2.19(\mathrm{~m}, 3 \mathrm{H})$, 2.16-1.99 (m, 2H), 1.96-1.64 (m, 3H). ${ }^{13} \mathrm{C}$ NMR $(75$ $\left.\mathrm{MHz}, \mathrm{CDCl}_{3}\right)$ : diastereomer $\mathrm{OH}_{\mathrm{ax}}: \delta 217.9,170.0$, $137.7,129.1$ (2C), 124.5, 120.3 (2C), 78.5, 54.4, 53.2, $38.5,26.3,25.8,18.6$, diastereomer $\mathrm{OH}_{\mathrm{eq}}: \delta 216.9$, 169.9, 137.6, 129.1 (2C), 124.6, 120.3 (2C), 74.7, 55.2, 53.9, 35.6, 27.5, 26.7, 15.5. MS $\left(\mathrm{ES}^{+}\right): \mathrm{m} / \mathrm{z}$ $\left[(\mathrm{M}+\mathrm{Na})^{+}\right] \quad 282 ;$ HRMS $(\mathrm{ES}+): \mathrm{m} / \mathrm{z}$ calcd for $\left[\left(\mathrm{C}_{15} \mathrm{H}_{17} \mathrm{NO}_{3}+\mathrm{H}\right)^{+}\right]$: 260.1281; found: 260.1284 . 11c: this product was synthesized starting from $4 c(R$ =1-naphthyl, $51 \mathrm{mg}, 0.2 \mathrm{mmol}$ ) by adapting the above procedure. After full conversion of $\mathbf{4 c}$, the excess of acrolein was eliminated by reduced pressure (100 mbar), without evaporation of toluene. Then DBU was added and the reaction mixture was stirred at room temperature for 1 hour to afford 11c as a 1:1.2 mixture of diastereomers as a viscous liquid $(38 \mathrm{mg}$, $62 \%)$. $\mathrm{R} f\left(\mathrm{EA} / \mathrm{CH}_{2} \mathrm{Cl}_{2}, 2: 3\right)=0.30$; Chiral HPLC (Lux-Amylose-2, hexane $/ \mathrm{PrOH}=7: 3$, flow rate $=1.0$ $\mathrm{mL} / \mathrm{min}, \lambda=254 \mathrm{~nm})$ : diastereomer $\mathrm{OH}_{\mathrm{ax}}: \mathrm{t}_{\text {minor }}=10.2$ min, $\mathrm{t}_{\text {major }}=33.5 \mathrm{~min}$, er $=7.5: 1$, diastereomer $\mathrm{OH}_{\mathrm{eq}}$ : $\mathrm{t}_{\text {minor }}=6.9 \mathrm{~min}, \mathrm{t}_{\text {major }}=7.9 \mathrm{~min}$, er $=8.0: 1 ;{ }^{1} \mathrm{H} \mathrm{NMR}$ $\left(400 \mathrm{MHz}, \mathrm{CDCl}_{3}\right)$ : diastereomer $\mathrm{OH}_{\mathrm{ax}}: \delta 10.38(\mathrm{~s}$, $\mathrm{NH}), 8.18-8.12(\mathrm{~m}, 2 \mathrm{H}), 7.86(\mathrm{~d}, J=7.7 \mathrm{~Hz}, 1 \mathrm{H}), 7.67$ $(\mathrm{d}, J=8.2 \mathrm{~Hz}, 1 \mathrm{H}), 7.61-7.55(\mathrm{~m}, 1 \mathrm{H}), 7.54-7.44(\mathrm{~m}$, 2H), 4.41-4.30 (m, 1H), $2.64(\mathrm{dd}, J=7.1,5.2 \mathrm{~Hz}, 1 \mathrm{H})$, 2.55-2.21 (m, 4H), 2.20-2.07 (m, 1H), 1.88-1.65 (m, $3 \mathrm{H})$, diastereomer $\mathrm{OH}_{\mathrm{eq}}: \delta 10.24(\mathrm{~s}, \mathrm{NH}), 8.18-8.12$ $(\mathrm{m}, 1 \mathrm{H}), 8.09$ (d, $J=8.4 \mathrm{~Hz}, 1 \mathrm{H}), 7.87(\mathrm{~d}, J=7.8 \mathrm{~Hz}$, $1 \mathrm{H}), 7.67(\mathrm{~d}, J=8.2 \mathrm{~Hz}, 1 \mathrm{H}), 7.61-7.55(\mathrm{~m}, 1 \mathrm{H})$, 7.54-7.44 (m, 2H), 4.17-4.04 (m, 1H), $2.76(\mathrm{dd}, J=$ 6.9, 3.2 Hz, 1H), 2.55-2.21 (m, 3H), 2.20-2.07 (m, 1H), 2.06-1.89 (m, 2H), 1.88-1.65 (m, 2H). ${ }^{13} \mathrm{C} \mathrm{NMR}$ $\left(75 \mathrm{MHz}, \mathrm{CDCl}_{3}\right)$ : diastereomer $\mathrm{OH}_{\mathrm{ax}}: \delta 170.5,134.2$, $132.6,128.8,126.6,126.5,126.1,125.8,125.3,120.9$, $119.3,78.7,54.9,53.2,38.9,26.4,25.9,18.7$, diastereomer $\mathrm{OH}_{\mathrm{eq}}: \delta 217.6,170.3,134.2,132.5$, $128.8,126.6,126.5,126.1,125.8,125.3,120.7,119.4$, 74.8, 55.2, 54.4, 35.9, 27.6, 26.8, 15.6. MS $\left(\mathrm{ES}^{+}\right): \mathrm{m} / \mathrm{z}$ $\left[(\mathrm{M}+\mathrm{Na})^{+}\right] \quad 332$; HRMS (ES+): $\mathrm{m} / \mathrm{z}$ calcd for $\left[\left(\mathrm{C}_{19} \mathrm{H}_{19} \mathrm{NO}_{3}+\mathrm{H}\right)^{+}\right]$: 310.1438 ; found: 310.1443 .

11d: this product was synthesized starting from $4 d(R$ $=\mathrm{Ts}, 56 \mathrm{mg}, 0.2 \mathrm{mmol})$ by adapting the above procedure. After addition of $\mathrm{DBU}$, the reaction mixture was heated at $60{ }^{\circ} \mathrm{C}$ for 6 hours to afford 11d as a 1:1 mixture of diastereomers as a viscous liquid (33 mg, 49\%). $\mathrm{R} f\left(\mathrm{EA} / \mathrm{CH}_{2} \mathrm{Cl}_{2}, 2: 3\right)=0.55$; Chiral HPLC (Chiralpak IC, hexane $/ i \mathrm{PrOH} / \mathrm{TFA}=7: 3: 0.01$, flow rate $=1.0 \mathrm{~mL} / \mathrm{min}, \lambda=254 \mathrm{~nm})$ : diastereomer $\mathrm{OH}_{\mathrm{ax}}: \mathrm{t}_{\text {minor }}=45.6 \mathrm{~min}, \mathrm{t}_{\text {major }}=48.3 \mathrm{~min}$, er $=66: 1$, diastereomer $\mathrm{OH}_{\mathrm{eq}}: \mathrm{t}_{\text {minor }}=26.6 \mathrm{~min}, \mathrm{t}_{\text {major }}=32.6 \mathrm{~min}$, er $=14: 1 ;{ }^{1} \mathrm{H} \mathrm{NMR}\left(400 \mathrm{MHz}, \mathrm{CDCl}_{3}\right)$ : diastereomer $\mathrm{OH}_{\mathrm{ax}}: \delta 10.23(\mathrm{~s}, \mathrm{NH}), 7.93(\mathrm{~d}, J=8.4,2 \mathrm{H}), 7.31(\mathrm{~d}, J$ $=8.4 \mathrm{~Hz}, 2 \mathrm{H}), 4.36(\mathrm{~d}, J=1.7 \mathrm{~Hz}, 1 \mathrm{H}), 2.60(\mathrm{dd}, J=$ 6.9, $5.5 \mathrm{~Hz}, 1 \mathrm{H}), 2.42(\mathrm{~s}, 3 \mathrm{H}), 2.35-2.26(\mathrm{~m}, 1 \mathrm{H})$, 2.15-1.98 (m, 4H), 1.92-1.64 (m, 3H), diastereomer $\mathrm{OH}_{\mathrm{eq}}: \delta 10.12(\mathrm{~s}, \mathrm{NH}), 7.93(\mathrm{~d}, J=8.4,2 \mathrm{H}), 7.31(\mathrm{~d}, J$ $=8.4 \mathrm{~Hz}, 2 \mathrm{H}), 4.10-4.07(\mathrm{~m}, 1 \mathrm{H}), 2.70(\mathrm{dd}, J=6.9$, $3.2 \mathrm{~Hz}, 1 \mathrm{H}), 2.42(\mathrm{~s}, 3 \mathrm{H}), 2.35-2.26(\mathrm{~m}, 1 \mathrm{H}), 2.15-$ 1.98 (m, 4H), 1.92-1.64 (m, 3H). ${ }^{13} \mathrm{C}$ NMR (75 MHz, $\left.\mathrm{CDCl}_{3}\right)$ : diastereomer $\mathrm{OH}_{\mathrm{ax}}: \delta 216.0,169.4,145.1$, $135.8,129.7$ (2C), 128.5 (2C), 78.2, 55.5, 52.5, 37.9, $26.4,25.5,21.8,18.6$, diastereomer $\mathrm{OH}_{\mathrm{eq}}: \delta 215.1$, $169.4,145.2,135.7,129.7$ (2C), 128.5 (2C), 74.4, 55.1, 54.5, 35.1, 26.6, 25.4, 21.8, 15.6. HRMS (ES+): 
$\mathrm{m} / \mathrm{z}$ calcd for $\left[\left(\mathrm{C}_{16} \mathrm{H}_{19} \mathrm{NO}_{5} \mathrm{~S}+\mathrm{H}\right)^{+}\right]: 338.1057$; found: 338.1061 .

12: a solution of 11d $(0.2 \mathrm{mmol}, 1$ equiv. $)$, and 2 iodoxybenzoic acid (IBX) $(0.4 \mathrm{mmol}, 2$ equiv) in ethyl acetate $(1 \mathrm{~mL})$ was refluxed in a sealed tube for 18 hours. After cooling to room temperature, the mixture was filtered through a short pad of Celite ${ }^{\circledR}$ and was concentrated under reduced pressure to afford quantitatively the crude ketone 12, which could not be further purified because of its instability on silica gel. $\mathrm{R} f(\mathrm{EA} / \mathrm{PE}, 1: 3)=0.22$; Chiral HPLC (Chiralpak IC, hexane $/$ EtOH $/$ TFA $=8: 2: 0.01$, flow rate $=1.0$ $\mathrm{mL} / \mathrm{min}, \lambda=254 \mathrm{~nm}): \mathrm{t}_{\text {major }}=12.1 \mathrm{~min}, \mathrm{t}_{\text {minor }}=13.9$ min, er $=24: 1 ;{ }^{1} \mathrm{H}$ NMR $\left(400 \mathrm{MHz}, \mathrm{CDCl}_{3}\right): \delta 7.94$ (d, $J=8.3 \mathrm{~Hz}, 2 \mathrm{H}), 7.32$ (d, $J=8.3 \mathrm{~Hz}, 2 \mathrm{H}), 3.35$ (d, $J$ $=6.8 \mathrm{~Hz}, 1 \mathrm{H}), 2.79(\mathrm{ddd}, J=17.0,12.6,9.4 \mathrm{~Hz}, 1 \mathrm{H})$, $2.50(\mathrm{dd}, J=17.0,6.7 \mathrm{~Hz}, 1 \mathrm{H}), 2.45-2.37(\mathrm{~m}, 1 \mathrm{H})$, 2.42 (s, 3H), 2.28-2.10 (m, 3H), 1.97 (ddd, $J=14.3$, 8.0, 3.1 Hz, $1 \mathrm{H}), 1.84-1.73(\mathrm{~m}, 1 \mathrm{H}) .{ }^{13} \mathrm{C}$ NMR $(75$ $\left.\mathrm{MHz}, \mathrm{CDCl}_{3}\right): \delta 206.0,203.5,168.3,145.3,135.5$, 129.7 (2C), 128.5 (2C), 64.9, 56.1, 33.6, 30.4, 26.2, 21.8, 21.2. MS $\left(\mathrm{ES}^{+}\right): \mathrm{m} / \mathrm{z}\left[(\mathrm{M}+\mathrm{Na})^{+}\right]$358; HRMS $(\mathrm{ES}+): \mathrm{m} / \mathrm{z}$ calcd for $\left[\left(\mathrm{C}_{16} \mathrm{H}_{17} \mathrm{NO}_{5} \mathrm{~S}+\mathrm{H}\right)^{+}\right]: 336.0900$; found: 336.0898 .

\section{Acknowledgements}

The Agence Nationale pour la Recherche (ANR-11-BS070014), the Centre National de la Recherche Scientifique (CNRS) and the Aix-Marseille Université are gratefully acknowledged for financial support.

Supporting Information for this article contains copies of ${ }^{1} \mathrm{H}$ and ${ }^{13} \mathrm{C}$ NMR spectra of all new compounds, and is available online at http://www.thieme-connect.de/ejournals/toc/synthesis.

\section{References}

\section{Abstract :}

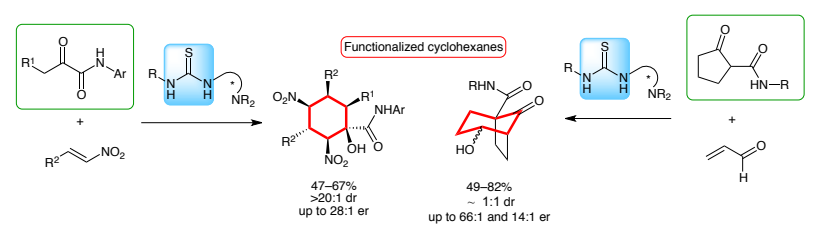


(1) For reviews on organocatalytic domino reactions, see: (a) Enders, D.; Grondal, C.; Hüttl, M. R. M. Angew. Chem. Int. Ed. 2007, 46, 1570. (b) Yu, X.; Wang, W. Org. Biomol. Chem. 2008, 6, 2037. (c) Alba, A.-N.; Companyo, X.; Viciano, M.; Rios, R. Curr. Org. Chem. 2009, 13, 1432. (d) Grondal, C.; Jeanty, M.; Enders, D. Nature Chem. 2010, 2, 167. (e) Pellisier, H. Adv. Synth. Catal. 2012, 354, 237.

(2) Li, J. J. (ed) (2010) Six-Membered Carbocycles, in Name Reactions for Carbocyclic Ring Formations, John Wiley \& Sons, Inc., Hoboken, NJ, USA.

(3) For selected examples of two-component approaches, see: (a) Halland, N.; Aburel, S. P; Jorgensen, K. A. Angew. Chem. Int. Ed. 2004, 43, 1272. (b) Zhou, J.; List, B. J. Am. Chem. Soc. 2007, 129, 7498. (c) Reyes, E.; Jiang, H.; Milelli, A.; Elsner, P.; Hazell, R. G.; Jørgensen, K. A. Angew. Chem. Int. Ed. 2007, 46, 902. (d) McGarraugh, P. G.; Brenner, S. E. Org. Lett. 2009, 11, 5654. (e) Hayashi, Y.; Okano, T.; Aratake, S.; Hazelard, D. Angew. Chem. Int. Ed. 2007, 46, 4922. (f) Anwar, S.; Chang, H.-J.; Chen, K. Org. Lett. 2011, 13, 2200.

(4) For selected examples of multicomponent approaches, see: (a) Ramachary, D. B.; Chowdari, N. S.; Barbas, C. F., III Angew. Chem. Int. Ed. 2003, 42, 4233. (b) Enders, D.; Hüttl, M. R. M.; Runsik, Y.; Raabe, G.; Wendt, B. Angew. Chem. Int. Ed. 2007, 46, 467. (c) Penon, O.; Carlone, A.; Mazzanti, A.; Locatelli, M. Sambri, L,; Bartoli, G.; Melchiorre, P. Chem. Eur. J. 2008, 14, 4788. (d) Wang, Y.; Han, R. G.; Zhao, Y. L.; Yang, S.; Xu, P. F.; Dixon, D. J. Angew. Chem. Int. Ed. 2009, 48, 9834. (e) Jiang, K.; Jia, Z.-J.; Yin, X.; Wu, L.; Chen, Y.-C. Org. Lett. 2010, 12, 2766. (f) Jia, Y.; Mao, Z.; Wang, R. Tetrahedron: Asymmetry 2011, 22, 2018.

(5) For a concept on this subject, see: Bonne, D.; Constantieux, T.; Coquerel, Y.; Rodriguez, J. Chem. Eur. J. 2013, 19, 2218. For a review, see: Bonne, D.; Constantieux, T.; Coquerel, Y.; Rodriguez, J. Tetrahedron: Asymmetry 2010, $21,1085$.

(6) (a) Baslé, O.; Raimondi, W.; Sanchez Duque, M. M.; Bonne, D.; Constantieux, T.; Rodriguez., J. Org. Lett. 2010, 12, 5246. (b) Sanchez Duque, M. M.; Baslé, O.; Isambert, N.; Gaudel-Siri, A.; Génisson, Y.; Plaquevent, J.-C.; Rodriguez, J.; Constantieux, T. Org. Lett. 2011, 13, 3296.

(7) For similar sequences with 1,2-ketoesters with a chiral copper(II) complex, see: (a) Nakamura, A.; Lectard, S.; Hashizume, D.; Hamashima, Y.; Sodeoka, M. J. Am. Chem. Soc., 2010, 132, 4036. (b) Shi, D.; Xie, Y.; Zhou, H.; Xia, C.; Huang, H. Angew. Chem. Int. Ed. 2012, 51, 1248. For a related three-component one-pot sequence, see: (c) Varga, S.; Jakab, G.; Drahos, L.; Holczbauer, T.; Czugler, M.; Soós, T. Org. Lett. 2011, 13, 5416.

(8) Okino, T.; Hoashi, Y.; Furukawa, T.; Xu, X.; Takemoto, Y. J. Am. Chem. Soc. 2005, 127, 119.

(9) For recent reviews, see : Presset, M.; Coquerel, Y., Rodriguez, J. ChemCatChem 2012, 4, 172. Presset, M.; Coquerel, Y., Rodriguez, J. Chem. Rev. 2013, 113, 525.

(10) For examples of $N$-heterocyclic carbene-catalyzed enolization and Michael additions, see: (a) Boddaert, T.; Coquerel, Y.; Rodriguez, J. Adv. Synth. Catal. 2009, 351, 1744. (b) Boddaert, T.; Coquerel, Y.; Rodriguez, J. Chem. Eur. J. 2011, 17, 2266.

(11) 1,3-Ketoamides were prepared following : Presset, M.; Coquerel, Y.; Rodriguez, J. J. Org. Chem. 2009, 74, 415.

(12) HPLC analysis of the corresponding hemiaminal 10d, after oxidation, showed that it was obtained with 84:1 er. See reference $6 \mathrm{~b}$ for details.

(13) For reviews on the use of 1,2-dicarbonyls as pronucleophiles, see: Raimondi, W.; Bonne, D.; Rodriguez, J. Angew. Chem. Int. Ed. 2012 , 51, 40. (b) Raimondi, W.; Bonne, D.; Rodriguez, J. Chem. Commun. 2012 , 48, 6763. 